\title{
Ray Theory Analysis of the Shadow Blister Effect
}

James A. Lock

Cleveland State University, j.lock@csuohio.edu

Follow this and additional works at: https://engagedscholarship.csuohio.edu/sciphysics_facpub

Part of the Physics Commons

How does access to this work benefit you? Let us know!

\section{Publisher's Statement}

This paper was published in Applied Optics and is made available as an electronic reprint with the permission of OSA. The paper can be found at the following URL on the OSA website: http://www.opticsinfobase.org/ao/abstract.cfm?URI=ao-38-9-1573. Systematic or multiple reproduction or distribution to multiple locations via electronic or other means is prohibited and is subject to penalties under law.

\section{Original Citation}

Lock, James A. "Ray Theory Analysis of the Shadow Blister Effect." Applied Optics 37 (1998): 1573-1578.

\section{Repository Citation}

Lock, James A., "Ray Theory Analysis of the Shadow Blister Effect" (1998). Physics Faculty Publications. 76.

https://engagedscholarship.csuohio.edu/sciphysics_facpub/76

This Article is brought to you for free and open access by the Physics Department at EngagedScholarship@CSU. It has been accepted for inclusion in Physics Faculty Publications by an authorized administrator of

EngagedScholarship@CSU. For more information, please contact library.es@csuohio.edu. 


\title{
Ray theory analysis of the shadow blister effect
}

\author{
James A. Lock
}

\begin{abstract}
When an extended light source such as the Sun illuminates two objects so that their shadows lie close to each other, the shadow of one of the objects occasionally appears to bulge out toward the shadow of the other. This effect is caused by the overlap of the penumbras of the shadows and is analyzed here with ray theory. A laboratory demonstration of this phenomenon is performed and compared with theoretical predictions. (C) 1998 Optical Society of America
\end{abstract}

OCIS code: 080.0080.

\section{Introduction}

A number of optical phenomena involving shadows of objects illuminated by the Sun are described in the books by Minnaert ${ }^{1}$ and by Lynch and Livingston. ${ }^{2}$ Another interesting shadow phenomenon not mentioned there, which is similar to the blister effect for misfocused vision, ${ }^{3,4}$ is described as follows. Consider the shadows on a viewing screen of two objects $\alpha$ and $\beta$ illuminated by the Sun or some other extended light source. Because of the angular extent of the source, each shadow has both umbra and penumbra regions. If $\alpha$ is closer to the viewing screen than $\beta$ is, its penumbra is narrower than that of $\beta$. If one of the objects is moved so that the penumbras of the two shadows overlap, the umbra of $\alpha$ appears to bulge out toward the umbra of $\beta$, giving a blisterlike appearance to the umbra of $\alpha$. This is the shadow blister effect.

This phenomenon may be easily observed with illumination by the Sun. For example, consider sunlight entering a room through a window when the Sun is relatively low in the sky. If a person stands in the middle of the room and moves his or her head until the shadow of the head on the wall opposite the window almost touches the edge of the shadow of the window frame, the shadow of the person's head appears to bulge toward the shadow of the window frame. A similar bulging can be observed outdoors if a person stands a few meters from a tree trunk or the edge of a wall on a sunny day and moves his or her

The author is with the Department of Physics, Cleveland State University, Cleveland, Ohio 44115.

Received 16 June 1997; revised manuscript received 15 October 1997.

0003-6935/98/091573-06\$15.00/0

(C) 1998 Optical Society of America head until the shadow of the head almost touches the shadow of the tree trunk or wall on the ground. The size of the shadow bulge depends, among other things, on the distance between the tree or the wall and the observer's head and on the distance between the observer's head and the location on the ground where the shadows lie. Thus some experimentation with these distances is necessary in order to produce a visually apparent shadow blister.

\section{Ray Theory Model of the Shadow Blister Effect}

\section{A. Line-Source and Straight-Edge Obstacles}

The shadow blister effect may be physically understood in terms of ray optics. In order to demonstrate why it occurs, we examine first a simple geometry, and once the physical mechanism of the effect is understood in this context, we examine more complicated geometries. We consider the shadows of two long straight parallel walls illuminated by a distant one-dimensional line source of light oriented perpendicularly to the edges of the walls, as is shown in Fig. 1. The incoherent line source has length $D$ and infinitesimal height. The objects $\beta$ and $\alpha$ (in this case the two walls) are distances $L$ and $L+x$ away from it, respectively. The shadows of $\alpha$ and $\beta$ fall on a viewing screen located a distance $R$ from $\alpha$ and placed parallel to the two walls. The transverse separation of $\alpha$ and $\beta$ is $a$, and the horizontal and the vertical viewing screen coordinates are $y_{d}$ and $z_{d}$, respectively. Both the edge of $\beta$ and the origin of the viewing screen coordinate system are in line with the center of the source. Object $\beta$ is considered to be fixed, and changes in the transverse separation of $\alpha$ and $\beta$ are accomplished when $\alpha$ is moved.

The light intensity at any viewing screen position $\left(y_{d}, z_{d}\right)$ is proportional to the fraction of the length of the line source unobscured at that location by $\alpha$ and 


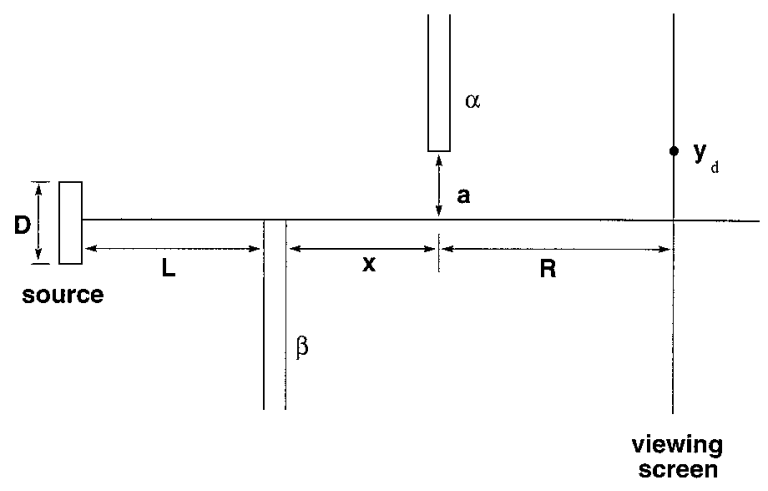

Fig. 1. Two objects $\alpha$ and $\beta$ at different locations between a onedimensional line source of light and the viewing screen. Both the edge of $\beta$ and the origin of the viewing screen coordinate system are in line with the center of the source. The coordinate $y_{d}$ on the viewing screen is in the plane of the figure, and $z_{d}$ is perpendicular to the plane of the figure.

$\beta$, neglecting the obliquity factors of the rays. In order to simplify the resulting equations algebraically, the scaled transverse separation of $\alpha$ and $\beta$,

$$
A=2 a L / D,
$$

and the scaled horizontal viewing screen coordinate,

$$
Y=2 y_{d} L / D,
$$

are used. The two scaled distances of Eqs. (1) and (2) are the actual distances divided by the angular radius of the source at $\beta$. For simplicity, we also assume that $L \gg x$ and $L \gg R$, which correspond to a distant source, although the shadow blister effect also occurs if the source is not distant.

A straightforward analysis of the geometry of Fig. 1 distinguishes three cases for the scaled transverse separation $A$. Case 1 corresponds to a large transverse separation of $\alpha$ and $\beta$ with $A \geq x+2 R$. For this case the penumbras of the two shadows do not overlap. We then have, reading from positive $Y$ (or $y_{d}$ ) on the viewing screen [Eq. (3a)] to negative $Y$ [Eq. $(3 \mathrm{e})$,

$$
\begin{array}{ll}
I(Y)=0 & \text { if } A+R \leq Y, \\
I(Y) \approx I_{0}[1+(A-Y) / R] / 2 & \\
I(Y)=I_{0} & \text { if } A-R \leq Y \leq A+R, \\
I(Y) \approx I_{0}[1+Y /(x+R)] / 2 & \text { if } x+R \leq Y \leq A-R, \\
I(Y)=0 & \text { if }-x-R \leq Y \leq x+R, \\
& \text { if } Y \leq-x-R,
\end{array}
$$

where the light intensity on the viewing screen in the absence of shadowing by the objects is $I_{0}$. Again neglecting the obliquity factors of the rays, the intensity is independent of $z_{d}$. Case 2 corresponds to a small transverse separation of $\alpha$ and $\beta$ with $A$ in the range $x \leq A \leq x+2 R$, where the two penumbras now overlap. For this case we have

$$
\begin{array}{ll}
I(Y)=0 & \text { if } A+R \leq Y, \\
I(Y) \approx I_{0}[1+(A-Y) / R] / 2 & \\
& \text { if } x+R \leq Y \leq A+R, \\
I(Y) \approx I_{0}[A / R-x Y / R(x+R)] / 2 & \\
I(Y) \approx I_{0}[1+Y /(x+R)] / 2 \\
\\
\text { if } A-R \leq Y \leq x+R, \\
I(Y)=0 & \text { if }-x-R \leq Y \leq A-R,
\end{array}
$$

Last, case 3 corresponds to transversely overlapping or nearly overlapping objects with $A$ in the range $-x \leq A \leq x$. For this case we have

$$
\begin{array}{ll}
I(Y)=0 & \text { if } \quad(x+R) A / x \leq Y, \\
I(Y) \approx I_{0}[A / R-x Y / R(x+R)] / 2 & \\
I(Y) \approx I_{0}[1+Y /(x+R)] / 2 \\
& \text { if } \quad A-R \leq Y \leq(x+R) A / x, \\
I(Y)=0 \quad \text { if } \quad-x-R \leq Y \leq A-R, \\
& \text { if } \quad Y \leq-x-R .
\end{array}
$$

Equations (3a), (4a), and (5a) describe the umbra of $\alpha$, and approximations (3b) and (4b) describe its penumbra. Similarly, Eqs. (3e), (4e), and (5d) describe the umbra of $\beta$, and approximations (3d), (4d), and (5c) describe its penumbra. Equation (3c) is the fully illuminated region between the edges of the two penumbras when $\alpha$ and $\beta$ are widely separated. Finally, approximations (4c) and (5b) describe the region of penumbra overlap. The scaled position on the viewing screen of the edges of the umbras and the penumbras of $\alpha$ and $\beta$ for cases 1,2 , and 3 is shown in Fig. 2 as a function of the scaled transverse separation $A$ for $x / R=1.0$. The slope of the uppermost diagonal line in Fig. 2 (which marks the boundary between the umbra and the penumbra of $\alpha$ for cases 1 and 2) is the rate of approach on the viewing screen of the umbra of $\alpha$ toward that of $\beta$ when the transverse separation of $\alpha$ and $\beta$ is decreased by keeping $\beta$ fixed and moving $\alpha$. In the region of transverse overlap or near overlap of $\alpha$ and $\beta$ (case 3), the umbra of $\alpha$ approaches that of $\beta$ at a more rapid rate when $A$ is decreased than is the case when $\alpha$ and $\beta$ are more widely transversely separated, as in cases 1 and 2 (i.e., the slope of the leftmost diagonal line in Fig. 2 is greater than the slope of the uppermost diagonal line). This more rapid approach gives the visual appearance of a protrusion from $\alpha$, which is the shadow blister.

The more rapid approach for case 3 is most visually apparent when the longitudinal separation of the two objects is moderate, i.e., when $x / R \approx O(1)$. An optimization of the visual appearance of the shadow blister is expected to occur because, on the one hand, if the two objects are the same distance from the viewing screen, i.e., $x / R=0$, the effect does not occur. 


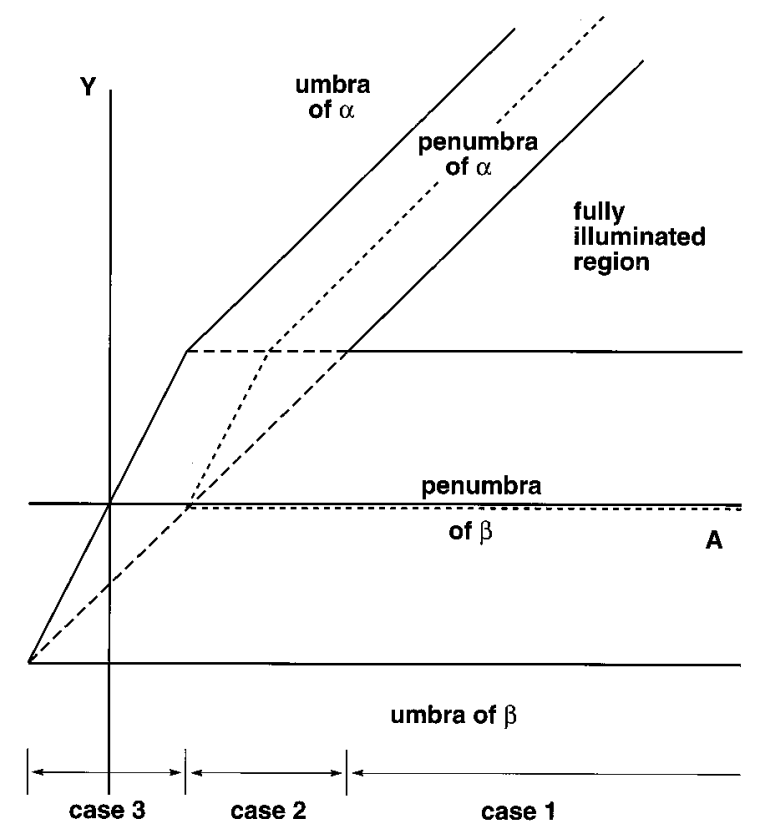

Fig. 2. Scaled positions on the viewing screen as defined in Eq. (2) of the edges of the umbras and penumbras of $\alpha$ and $\beta$ as a function of their scaled transverse separation as defined in Eq. (1) for the longitudinal separation $x=R$. The finely dashed lines correspond to the intensity $I_{0} / 2$, where $I_{0}$ is the intensity in the fully illuminated region. The two slightly coarser dashed lines mark the boundaries of the region of penumbra overlap.

This is shown in Fig. 3. In this situation, the intensity in the region of penumbra overlap is independent of the scaled viewing screen position for a given scaled transverse separation of $\alpha$ and $\beta$. On the other hand, when the longitudinal separation of $\alpha$ and $\beta$ is large, i.e., $x / R \gg 1$, the more rapid approach of the umbra of $\alpha$ toward that of $\beta$ in the region of penumbra overlap loses its visual impact, as the penumbra of $\alpha$ is quite narrow. The $x / R \approx O(1)$ condition is easily satisfied in the observation of the effect indoors by use of the solar illumination described in Section 1 if one stands approximately in the middle of the room between the window and the opposite wall.

The physical reason for the occurrence of the shadow blister effect may be simply understood by the following argument in the context of the distant line-source example. Consider first cases 1 or 2 , with an observer walking along the viewing screen from positive $y_{d}$ to negative $y_{d}$, as in Fig. 4 . The observer is first able to view a portion of the source at the location $y_{\alpha}$ when $\alpha$ ceases obscuring the source's lower edge. As the observer keeps walking, the light intensity first increases, reaches a maximum, and then decreases as a larger fraction of the source and then a smaller fraction are visible. Finally, the source ceases being visible at $y_{\beta}$ when $\beta$ obscures its top edge. The location $y_{\alpha}$ is the boundary between the umbra and the penumbra of $\alpha$, and $y_{\beta}$ is the boundary between the umbra and the penumbra of $\beta$.

Now consider an observer walking along the viewing screen from positive $y_{d}$ to negative $y_{d}$ for case 3 , as in Fig. 5. When $\alpha$ ceases obscuring the lower edge of

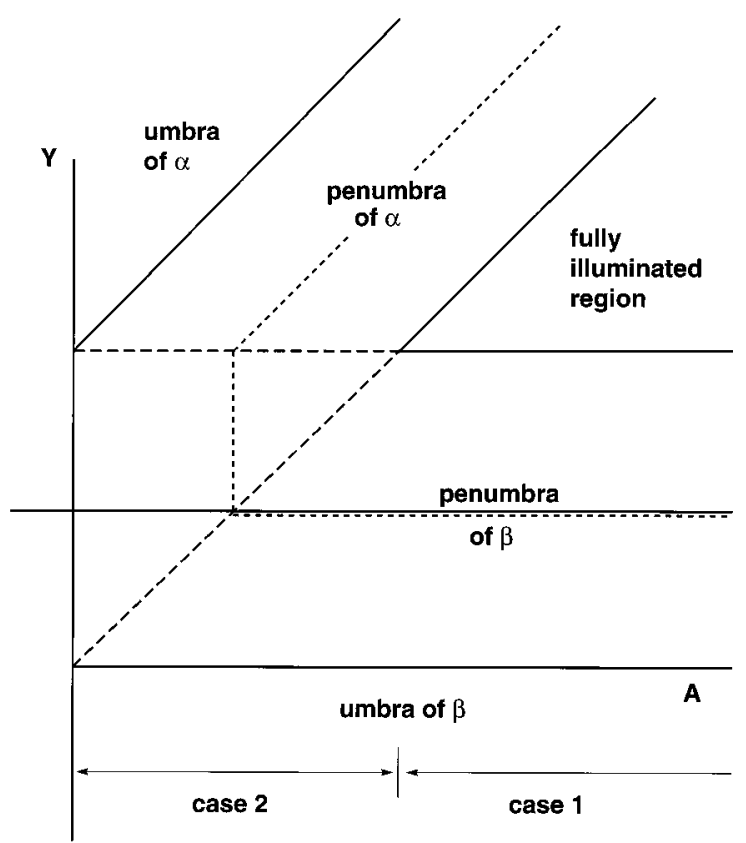

Fig. 3. Scaled positions on the viewing screen of the edges of the umbras and the penumbras of $\alpha$ and $\beta$ as functions of their scaled transverse separations when $\alpha$ and $\beta$ are the same distance from the viewing screen. The finely dashed lines correspond to the intensity $I_{0} / 2$. The two slightly coarser dashed lines mark the boundaries of the region of penumbra overlap.

the source at $y_{\alpha}$, the lower edge is still obscured by $\beta$. The observer must walk farther to $y_{\alpha}{ }^{\prime}$ until a point on the source is first unobscured by both $\alpha$ and $\beta$. The umbra of $\alpha$ thus occurs at a more negative viewing screen position in Fig. 5 for case 3 than it would based on an extrapolation of its position in Fig. 4 for cases 1 and 2. This is because the lower end of the source continues to be obscured by $\beta$ between $y_{\alpha}$ and $y_{\alpha}{ }^{\prime}$. This continued obscuration of the source by $\beta$ when the observer is past the position where the edge of the umbra of $\alpha$ would occur in the absence of $\beta$ is the cause of the apparent bulging of the shadow blister.

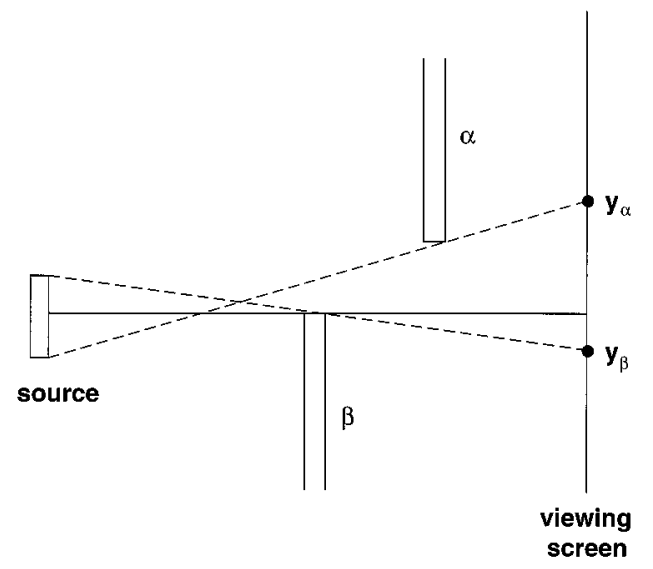

Fig. 4. Light rays at the edges of the umbras of $\alpha$ and $\beta$ for objects with a large transverse separation. 


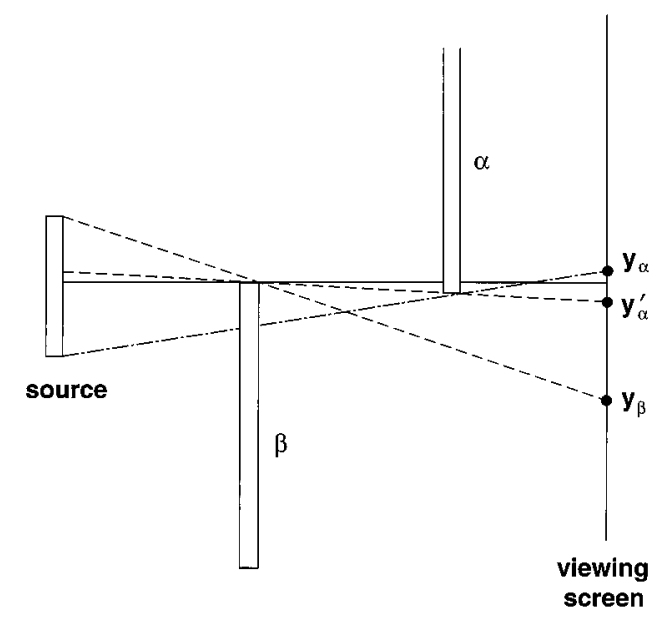

Fig. 5. Light rays at the edges of the umbras of $\alpha$ and $\beta$ for transversely overlapping objects. Object $\beta$ obscures the lower end of the source between $y_{\alpha}$ and $y_{\alpha}{ }^{\prime}$, thus extending the umbra of $\alpha$ and producing the shadow blister.

\section{B. Disk-Source and Straight-Edge Obstacles}

If a disk source of diameter $D$, rather than a line source, were used in Fig. 1 along with the two wall obstacles, the viewing screen intensity would again be proportional to the fraction of the disk source unobscured at a particular viewing screen position $\left(y_{d}\right.$, $z_{d}$ ). The unobscured fraction is most easily calculated when the edges of the long straight walls are projected onto the source plane from the viewing screen position in question. The qualitative behavior of the viewing screen intensity for a disk source is similar to that of Fig. 2 for a line source. But the intensity within the penumbra of $\beta$, for example, is now given by

$$
\begin{aligned}
I(Y)= & I_{0}-I_{0}\{\arccos [Y /(x+R)] \\
& \left.-Y\left[1-Y^{2} /(x+R)^{2}\right]^{1 / 2} /(x+R)\right\} / \pi
\end{aligned}
$$

for $-x-R \leq Y \leq x+R$. The intensity within the penumbra regions now varies quadratically as a function of $Y$ near the boundaries of each penumbra with both its respective umbra and with the fully illuminated region. To compensate for the slower intensity variation near the edges of the penumbras, the intensity near the center varies linearly as a function of $Y$, but with a larger slope than for the line-source case, in which the intensity varies linearly over the entire penumbra.

\section{One Disk Obstacle and One Straight-Edge Obstacle}

If either object $\alpha$ or $\beta$ is a sphere or disk, rather than a long straight wall, as is the case for the observations made with solar illumination described in Section 1, where $\beta$ is the observer's head, and the laboratory demonstration of Section 3, where it is a thin cardboard disk, the viewing screen intensity is again calculated in the same way. From each viewing screen position $\left(y_{d}, z_{d}\right)$, the edges of $\alpha$ and $\beta$ are projected back to the source plane and the fraction of either the disk source (for the observations of Section 1) or the line source (for the laboratory demonstration of Section 3) unobscured by the projections is calculated. The intensity at the viewing screen position is proportional to the unobscured fraction.

Consider, for example, a line source of length $D$, where object $\beta$ is a disk of radius $B$ and $\alpha$ is a long straight wall transversely offset by the distance $a$, as in Fig. 1. The edge of wall $\alpha$ and the edge of disk $\beta$ that is closer to $\alpha$ are projected from $\left(y_{d}, z_{d}\right)$ to the source plane and intersect the line source at

$$
\begin{aligned}
y_{\alpha s}= & a(L+x+R) / R-y_{d}(L+x) / R, \\
y_{\beta s}= & (L+x+R)\left\{-B+\left[B^{2}-z_{d}^{2} L^{2} /(L+x\right.\right. \\
& \left.\left.+R)^{2}\right]^{1 / 2}\right\} /(x+R)-y_{d} L /(x+R),
\end{aligned}
$$

where now it is not assumed that $L \gg x$ and $L \gg R$, as was the case in Eqs. (3)-(5). The intervals on the source plane $y \geq y_{\alpha s}$ and $y \leq y_{\beta s}$ are obscured by the projections of $\alpha$ and $\beta$, respectively. In the absence of $\beta$, the boundary on the viewing screen between the penumbra of $\alpha$ and the fully illuminated region is given by the straight line defined by Eq. (7) with $y_{\alpha s}$ $=D / 2$, and the boundary between the penumbra and the umbra of $\alpha$ is given by the straight line defined by $y_{\alpha s}=-D / 2$. Similarly, if $\alpha$ were absent, the boundary on the viewing screen between the penumbra the edge of $\beta$ that is closer to $\alpha$ and the fully illuminated region would be given by the half-circle defined in Eq. (8) with $y_{\beta s}=-D / 2$ and the boundary between the penumbra and the umbra of $\beta$ would be given by the half-circle defined by $y_{\beta s}=D / 2$. When both $\alpha$ and $\beta$ are present, the region of penumbra overlap corresponds to when the projections of $\alpha$ and $\beta$ each obscure a portion of the line source, i.e., $-D / 2 \leq y_{\beta s}<$ $y_{\alpha s} \leq D / 2$. The viewing screen intensity at $\left(y_{d}, z_{d}\right)$ in the penumbra overlap region is then

$$
I\left(y_{d}, z_{d}\right)=\left(y_{\alpha s}-y_{\beta s}\right) I_{0} / D .
$$

In the formation of the shadow blister in case 3 of Fig. 2, the umbra of $\beta$ touches the umbra of the shadow blister on $\alpha$ at the minimum transverse separation of $\alpha$ and $\beta$, i.e.,

$$
a=-D x / 2 L .
$$

At larger transverse separations in case 3,

$$
-D x / 2 L<a<D x / 2 L
$$

there is a penumbra gap between the umbra of $\beta$ and the umbra of the shadow blister on $\alpha$. In either case, the boundary between the penumbra and the umbra of the shadow blister occurs when the line source is totally obscured by the projections of $\alpha$ and $\beta$, i.e., when $y_{\beta s}=y_{\alpha s}$. Substitution of Eq. (10) into Eqs. (7) and (8) with the condition $y_{\beta s}=y_{\alpha s}$ gives the shape of the shadow blister on the umbra of $\alpha$ for the situation of the minimum transverse separation of $\alpha$ and $\beta$ and 


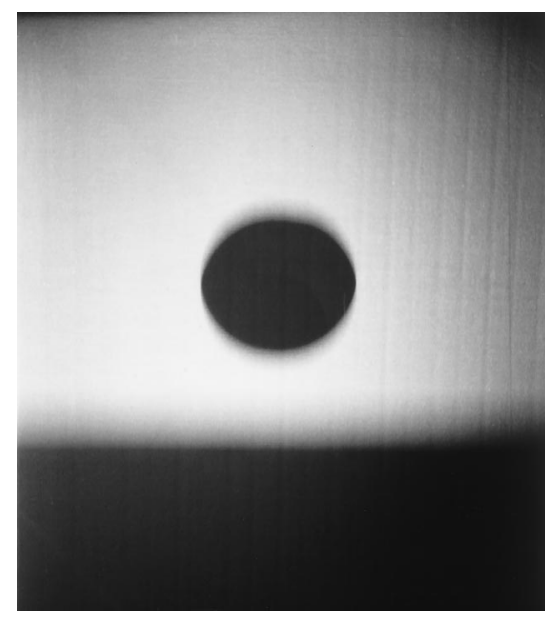

Fig. 6. Shadows of a thin circular cardboard disk of 13-cm diameter and a long wooden board with a straight edge illuminated by a tubular light bulb with a clear envelope and a 10-cm-long thin straight filament approximating a one-dimensional line source. The board and the disk have a wide transverse separation.

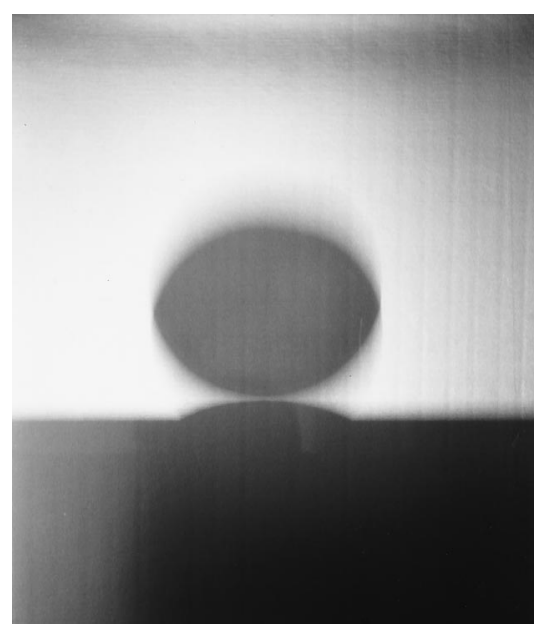

Fig. 7. Wooden board is closer to the viewing screen than the circular cardboard disk, and they transversely overlap. Thus the board's shadow appears to bulge toward the shadow of the disk.

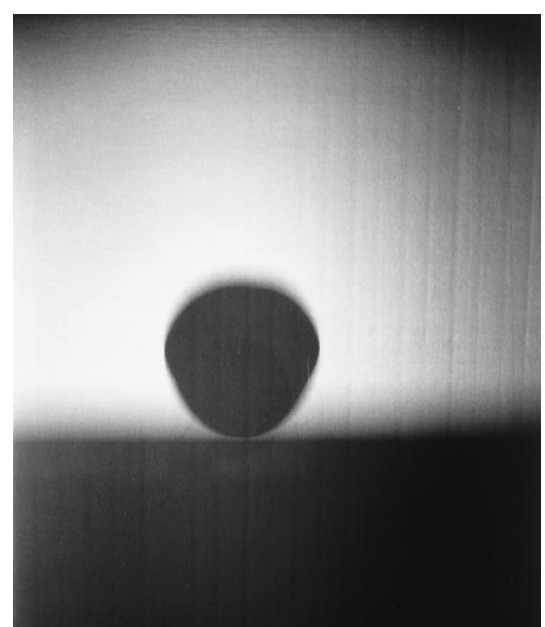

Fig. 8. Circular cardboard disk is closer to the viewing screen than the wooden board, and they transversely overlap. Thus the disk's shadow appears to bulge toward the shadow of the board. touching umbras. The shape is a section of the ellipse

$$
\begin{aligned}
x^{2}\left[(B R / x)-y_{d}-D(x\right. & +R) / 2 L]^{2} / R^{2} \\
& +z_{d}^{2} L^{2} /(L+x+R)^{2}=B^{2},
\end{aligned}
$$

and it bulges out from the long straight edges of the rest of the umbra of $\alpha$, which are given by

$$
y_{d}=-D(x+R) / 2 L+R D /(L+x) .
$$

The width of the shadow blister at $z_{d}=0$ is then

$$
\Delta y_{d}=R D /(L+x),
$$

and the blister intersects the long straight umbra of Eq. (13) at

$$
\begin{aligned}
z_{d \pm}= & \pm(L+x+R)[2 x B D /(L+x) \\
& \left.-x^{2} D^{2} /(L+x)^{2}\right]^{1 / 2} / L .
\end{aligned}
$$

The aspect ratio of the shadow blister is then

$$
\Delta y_{d} / \Delta z_{d}=\Delta y_{d} /\left(z_{d+}-z_{d_{-}}\right) .
$$

For a distant line source that subtends a small angle at the viewing screen, Eq. (16) simplifies to

$$
\Delta y_{d} / \Delta z_{d}=\left(D R^{2} / 2 L B x\right)^{1 / 2} .
$$

A similar calculation may be done for the larger transverse separations of case 3 with $a$ from inequality (11) substituted into Eqs. (7) and (8).

The shadow blister has its greatest visual impact when the aspect ratio of Eqs. (16) and (17) is large. This occurs when the angular radius of the source $D / 2 L$ is large, when the distance $R$ between $\alpha$ and the viewing screen is large, when the distance $x$ between $\alpha$ and $\beta$ is small, and when the radius $B$ of the disk obstacle is relatively small. These predictions are tested in the laboratory demonstration described in Section 3.

\section{Laboratory Demonstration}

To illustrate the shadow blister effect, the shadows of a long wooden board with a straight edge and a thin circular cardboard disk of diameter $2 B=13 \mathrm{~cm}$ illuminated by a $40-\mathrm{W}$ tubular light bulb with a clear glass envelope and a straight thin filament of length $D=10 \mathrm{~cm}$ were observed. This light source closely approximates the one-dimensional line source of Fig. 1. For each of the photographs of Figs. 6-8, the two objects were positioned so that $L=145 \mathrm{~cm}, x=70$ $\mathrm{cm}$, and $R=100 \mathrm{~cm}$. The distances $L, x$, and $R$ were chosen so that the size of the apparent protrusion on the umbra of $\alpha$ was approximately maximized. The angular diameter of the line source at the viewing screen was $1.8^{\circ}$, which is significantly larger than the $0.5^{\circ}$ angular diameter of the Sun. As was seen in Eq. (17), a larger angular diameter source produces wider penumbras and a more pronounced shadow blister than would be the case for solar illumination.

Figure 6 shows the shadows of the two objects when their transverse separation is large. The shadows consist of both umbra and penumbra regions. In Fig. 7 the wooden board with the straight edge was placed closer to the viewing screen (object $\alpha$ ), and its shadow appeared to bulge toward the shadow of the circular cardboard disk (object $\beta$ ), as predicted in Fig. 2. Correspondingly, when the cardboard disk was closer to the viewing screen (object $\alpha$ ) in Fig. 8, its shadow appeared to bulge toward the shadow of the wooden board (object $\beta$ ), also in agreement with Fig. 2.

For Fig. 7, in which the umbras of $\alpha$ and $\beta$ appeared to touch, the observed aspect ratio of the shadow 
blister on the umbra of the wooden board was compared with the theoretical prediction of Eq. (16). For the values of $L, x, R, D$, and $B$ quoted above, the predicted aspect ratio of the shadow blister at the condition of touching umbras is $\Delta y_{d} / \Delta z_{d}=0.125$. Determining the observed aspect ratio requires an estimate of the position of the boundary between the umbra and the penumbra of the shadow blister. The estimate was made as follows. The original photograph of Fig. 7 was photocopied so as both to amplify the contrast and to threshold the image. The shadow blister portion of the photograph was placed at the center of the object plane of the photocopy machine in order to minimize the effects of any aberrations that might be present in the optics of the machine. The contrast-amplified and thresholded image exhibited a sharp transition between the printed and the unprinted regions of the photocopy paper. This sharp transition was taken to be the boundary between the umbras and the penumbras. The aspect ratio of the shadow blister on the photocopy image was then measured to be $\Delta y_{d} / \Delta z_{d}=$ $0.125 \pm 0.005$, in excellent agreement with the prediction of Eq. (16).

\section{Discussion}

Determining the conditions for optimizing the shadow blister effect requires not only the physical mechanism illustrated in Figs. 4 and 5, but also an assessment of the role played by contrast-amplifying mechanisms in the human visual system, as well as information about the sizes of $\alpha$ and $\beta$. For example, the visual impact of the shadow blister effect is heightened by the fact that during casual viewing, people largely ignore penumbras and consider the full shadow of an object and its umbra to be identical.
Also, although the light intensity between the two nearly touching umbras in case 3 and in Figs. 7 and 8 is less than $I_{0}$, the visual perception of the light intensity there is that it is much brighter than its shadowed surroundings and is perhaps as bright as the fully illuminated regions some distance away. As for the sizes of the objects casting the shadows, the effect in Figs. 7 and 8 for fixed values of $D, x, L$, and $R$ was visually more impressive when the circular cardboard disk with a diameter of $13 \mathrm{~cm}$ was used than when another circular cardboard disk with a diameter of $26 \mathrm{~cm}$ was used. This is consistent with the predictions of Eqs. (16) and (17). In addition, because the size of the shadow blister is a larger fraction of the disk's diameter for the smaller disk, it acquires a greater subjective visual impact.

The explanation of the shadow blister effect presented here is qualitatively similar to the explanation of the blister effect for misfocused viewing of one's thumb and finger at different distances from one's eye. ${ }^{4}$ A difference, however, is that here the protrusion appears on the shadow of the object closer to the viewing screen whereas for the blister effect for misfocused vision, the protrusion appears on the finger farther from one's eye.

\section{References}

1. M. Minnaert, The Nature of Light and Color in the Open Air (Dover, New York, 1954), pp. 3-7.

2. D. K. Lynch and W. Livingston, Color and Light in Nature, (Cambridge U. Press, Cambridge, 1995), pp. 1-19.

3. F. M. Du Mas, "A new visual illusion," Am. J. Psychol. 66, 142-143 (1953).

4. J. R. Trotter, "The geometrical optics of the blister effect," Vision Res. 6, 587-596 (1966). 